\title{
Quantifying the impact of bacterial fitness and repeated antimicrobial exposure on the emergence of multidrug-resistant gram-negative bacilli
}

\author{
E.M.C. D’Agata ${ }^{a 1}$, M. Horn ${ }^{b}$ and G. Webb ${ }^{b}$ \\ ${ }^{a}$ Division of Infectious Diseases, Beth Israel Deaconess Medical Center \\ Harvard Medical School, Boston, MA, USA \\ ${ }^{b}$ Department of Mathematics, Vanderbilt University, Nashville, TN, USA
}

\begin{abstract}
The emergence of multidrug resistance among gram-negative bacilli is complex. Numerous factors need to be considered, including the biological fitness cost of resistance, fitnesscompensatory mutations and frequency and type of antibiotic exposure. A mathematical model evaluating these complex relationships was developed in an individual colonized with strains of pan-susceptible, single-, two- and multidrug-resistant (MDR) gram-negative bacilli (GN). The effect of bacterial fitness, compensatory mutations and the frequency of three-antimicrobial regimen exposure to predominance of multidrug-resistant strains were quantified. The model predicts that initially, in the absence of antibiotic exposure, the biologically fitter pan-susceptible strain predominates over the resistant strains. Over time, the fitness of the MDR strains increases faster with repeated antimicrobial exposure, through compensatory-fitness mutations. Increasing the frequency of exposure to the three-antimicrobial regimen or, increasing the initial fitness of the resistant strains, substantially decreases the time to MDR-GN predominance. The model implies that when MDR-GN strains evolve into strains that are fitter than susceptible strains, a reduction in antimicrobial exposure may not result in a decrease of MDR-GN, since the absence of selective antimicrobial pressure would no longer favor susceptible strains. The model also implies that antimicrobial cycling may promote the emergence of MDR-GN.
\end{abstract}

Key words: : multidrug-resistance, gram-negative bacteria, antibiotic resistance, mathematical modeling, bacterial fitness, antibiotic cycling

AMS subject classification: 92C50, 92C60

${ }^{1}$ Corresponding author. E-mail: edagata@bidmc.harvard.edu 


\section{Introduction}

The prevalence of antimicrobial-resistant bacteria continues to rise throughout the world [24]. Although methicillin-resistant Staphylococcus aureus (MRSA) and vancomycin-resistant enterococci (VRE) have been the major focus of investigations and preventive efforts, it has become clear over the last few years that the rate of hospital-acquired infections caused by multidrug-resistant gramnegative bacteria are quickly surpassing those caused by VRE and MRSA [9,13,15,16,24]. In one healthcare institution, MDR-P aeruginosa recovered form clinical cultures increased from $1 \%$ in 1994 to $16 \%$ in 2002, with similar dramatic increases among other species of gram-negative bacteria [9]. Effective antimicrobial therapy for infections caused by MDRGN is severely limited with over $30 \%$ of MDRGN resistant to 5 or more different antibiotic groups [28]. Understanding the interrelated and dynamic factors contributing to the emergence of MDRGN is crucial for the development of effective preventive strategies.

The human commensal flora provides the main ecological niche and reservoir for antimicrobialresistant gram-negative bacteria [37]. Although the majority of the gram-negative flora is comprised of antimicrobial-susceptible strains, subpopulations of resistant strains can exist through several mechanisms including spontaneous mutations, induction of resistance genes or exogenous acquisition of resistant strains [4,5,37]. The indirect effects of antimicrobial therapy targeting bacterial infections at other sites, can result in the overgrowth of these subpopulations of resistant strains with a concurrent decrease in bacterial concentrations of susceptible strains $[25,26]$.

During periods of endemicity, selective antimicrobial pressure is one of the major mechanism of MDRGN acquisition [11,27]. The endogenous acquisition of antimicrobial-resistant gramnegative bacteria implies that if a host is colonized with subpopulations of pansusceptible, single, two- and multidrug-resistant strains, sequential antimicrobial therapy with different antibiotics should promote the emergence of MDRGN in favor of the other strains of gram-negative bacteria (Figure 1). The frequency, patterns and types of antimicrobial exposure are therefore crucial to consider when analyzing the factors contributing to the emergence of MDRGN.

Another central factor to consider in understanding the emergence of antimicrobial-resistant bacteria is the deleterious effect of antimicrobial resistance genes on bacterial fitness. Resistance genes impose a biological fitness cost to bacteria by interfering with their physiological function, which can be expressed as decreased growth, survival or virulence [6,32]. Thus, in the absence of selective antibiotic pressure, the susceptible strains, which are fitter than the resistant ones, will predominate. Natural selection or compensatory evolution however, can result in mutations, which significantly reduce the cost of antimicrobial resistance and thereby increase the bacterial fitness of the resistant strain over time [1,10,21,23]. The potential evolution of MDRGN into strains which are fitter than pansusceptible ones, through these fitness-compensatory mutations, also needs to be considered in the analysis of factors contributing to the emergence of MDRGN, since these evolutionary processes would shift the competitive balance in favor of resistant strains even in the absence of antibiotic pressure.

A mathematical model of the population dynamics of single-drug resistant strains supports this hypothesis [20]. A mathematical model system was created to provide a framework describing the interrelated factors contributing to the emergence of MDRGN. In this model, multidrug-resistance 


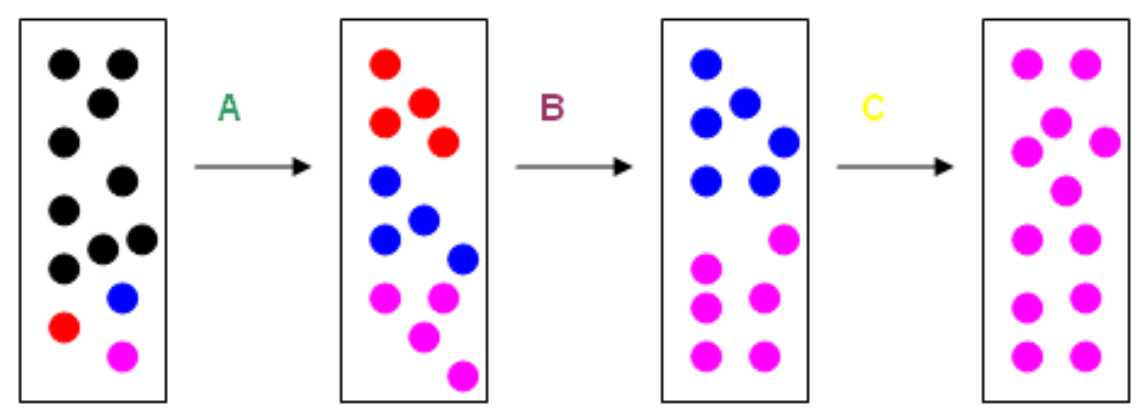

$A B C-S$

$A-R$ and $B C-S$

$A B-R$ and $C-S$

$A B C-R$

Figure 1: Diagram describing the emergence of multidrug-resistant bacteria, resistant to drugs A, B and $\mathrm{C}$ (ABCr-red), after antimicrobial exposure with drugs $\mathrm{A}, \mathrm{B}$ and $\mathrm{C}$. Four bacterial populations are present prior to antibiotic exposure: the pansensitive strains (ABCs-black) and subpopulations of single-drug resistant strains (resistant to drug A, and susceptible to drugs B and C [Ar-BCs-red]), two-drug resistant strains (resistant to drugs $\mathrm{A}$ and $\mathrm{B}$, and susceptible to drug $\mathrm{C}$, [ABr-Cs-blue]), and multidrug-resistant strains.

evolution was quantified at the level of the bacterial population within a single patient, since understanding the dynamics of resistance evolution at this level can provide important insights into the initial steps in the emergence and spread of MDRGN at the population level. It incorporates the impact of selective pressure from sequential and repeated exposure to multiple different antimicrobials and their quantitative and evolutionary effect on providing an advantage for antimicrobialresistant bacteria through fitness-compensatory mutations. The model assumes that, at baseline, an individual is colonized predominantly with pan-susceptible gram-negative bacteria and minute subpopulations of single-, double and MDRGN strains, and that the resistant strains are substantially less fit than the susceptible strains. Simulations are then performed to analyze the impact of changing the frequency of antimicrobial exposure to the time to emergence of MDRGN. The impact of the initial fitness cost of antimicrobial resistance and the effect of evolutionary compensatory mutations, which over time increase the fitness of the resistant strains, are also incorporated into the model.

\section{Baseline mathematical model}

The theoretical model consists of an individual patient who is harboring 4 strains of gram-negative bacteria. The four strains are ABCs (susceptible to antimicrobials A, B and C), Ar (resistant to antimicrobial $\mathrm{A}$ and susceptible to antimicrobials $\mathrm{B}$ and $\mathrm{C}$ ), $\mathrm{ABr}$ (resistant to $\mathrm{A}$ and $\mathrm{B}$, and susceptible to $\mathrm{C}$ ) and $\mathrm{ABCr}$ (resistant to all three antimicrobials). At time (0) the total bacterial population count is dominated by the pan-susceptible strain $\mathrm{ABCs}$, with very small subpopulations of $\mathrm{Ar}, \mathrm{ABr}$ and $\mathrm{ABCr}$ strains present. The model assumes that the constant minute source of 

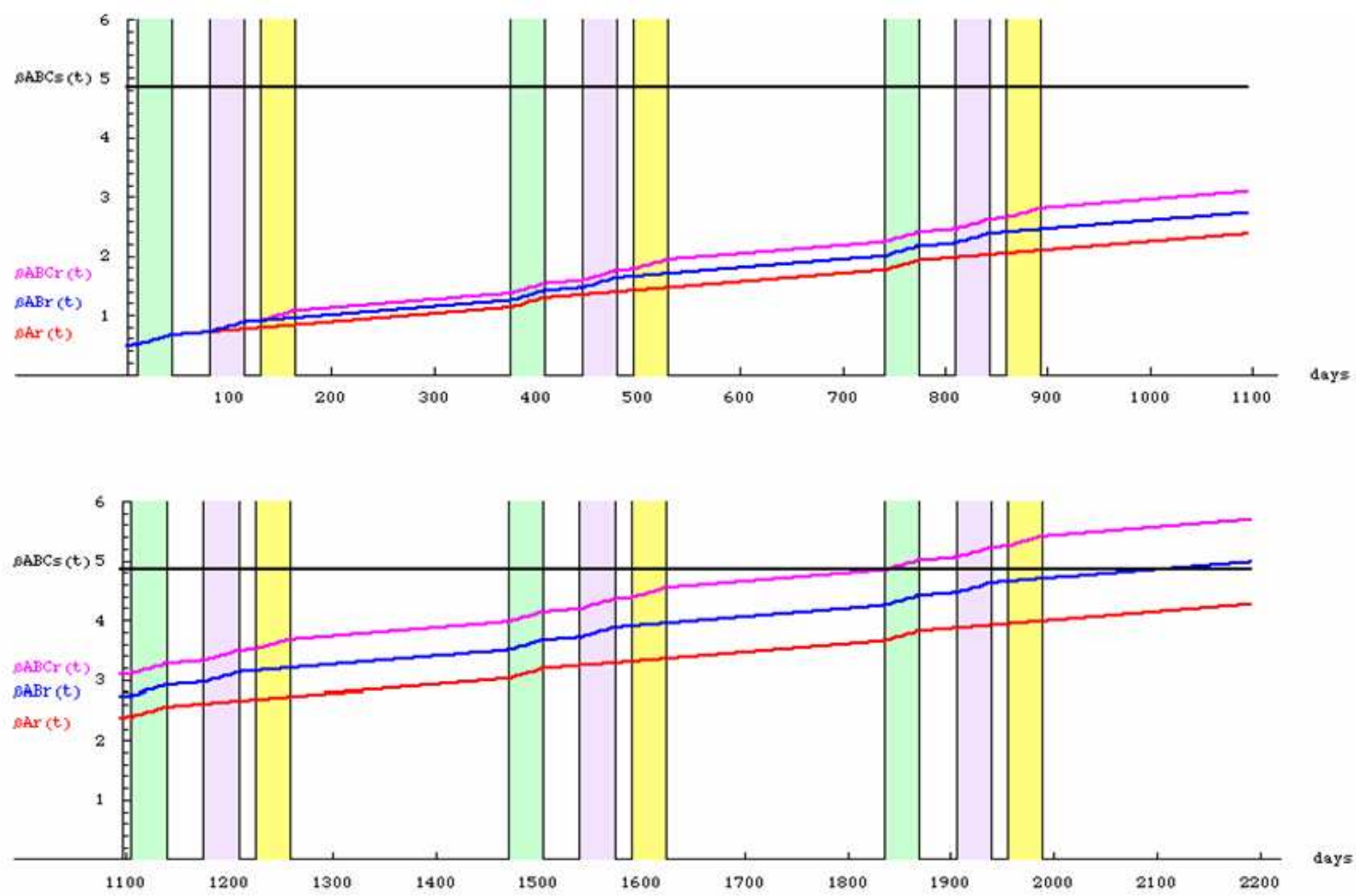

Figure 2: The fitness of 4 bacterial strains in the baseline simulation, $A B C s(t)$ (black), $r(t)$ (red), $A B r(t)$ (blue), and $A B C r(t)$ (magenta) as a function of time over 6 years. $\log 2 / \beta$ represents the doubling time of strains per day. Three antibiotic, A (green), B (purple), and C (yellow)] are administered every 12 months, with an initial difference in bacterial fitness between resistant strains and susceptible strains of $10 \%$. The fully resistant strain $A B C r$ has highest fitness at approximately 5 years. 
subpopulations of resistant strains arises through spontaneous mutations conferring resistance or exogenous acquisition of resistant strains [17]. There is a carrying capacity of the host defined as the population level that the host is capable of sustaining, or a saturation population density. The commensal flora is always at carrying capacity with the majority of the bacterial population represented by the pansusceptible, fitter strains in between treatments and by the resistant strains during therapy [31]. The intrinsic doubling time of the bacteria is independent of the carrying capacity; however, as the saturation population density is approached, fewer bacteria will survive to replicate [12].

The individual is then exposed to multiple 14-day courses of three different antimicrobials (A, B and C) every 12 months. During antimicrobial treatments, there is a loss of the appropriate antibiotic susceptible strain and an increase in the appropriate antibiotic resistant strain. The model assumes that the relative initial, or baseline, bacterial fitness of the resistant strains is $10 \%$ of the pan-susceptible strain. Over time, the fitness of the resistant strains increases at a rate of $2 x 10^{-4}$ per generation off antibiotics, through the evolution of compensatory mutations, and that during antibiotic exposure, the bacterial fitness of the appropriate antibiotic resistant strain increases faster since the rate of formation of compensatory mutations are proportional to the quantity of bacteria $[18,19]$. The bacterial fitness of ABCs remains constant throughout time (See Appendix).

Model simulations were performed to determine the effect of varying the frequency of exposure to the three-antimicrobial regimen from 12-month intervals to intervals ranging from 6 to 24months with a baseline fitness of $10 \%$ among the resistant strains relative to the pan-susceptible strains.

\section{Results}

\subsection{First cycle of exposure to three antimicrobials}

In the baseline simulation an individual patient is exposed to successive 14-day courses of three different antimicrobials (A, B and C) during a 12-month period. At time (0), before the initiation of antibiotic exposure, the pan-susceptible strain $\mathrm{ABCs}$ dominates over the 3 resistant strains due to its greater bacterial fitness. During antimicrobial treatments, there is an imposed loss of the appropriate susceptible strain due to selective action of the antimicrobials, accompanied by an increase in fitness of the 3 antimicrobial-resistant strains (Figure 2). During antimicrobial exposure the bacterial counts of the susceptible and resistant-strains decrease or increase appropriately. For example, the total bacterial count of $\mathrm{ABCs}$ (black line) decreases during exposure to antibiotic $\mathrm{A}$ (green bar) whereas the bacterial counts of the three resistant strains, all of which are resistant to antibiotic A, increase (Figure 3). After completion of the first cycle of antimicrobial exposure, the bacterial count of the $\mathrm{ABCs}$ and resistant strains return almost to their values at time (0), with the $\mathrm{ABCs}$ strain again dominating (Figure 3 ). 

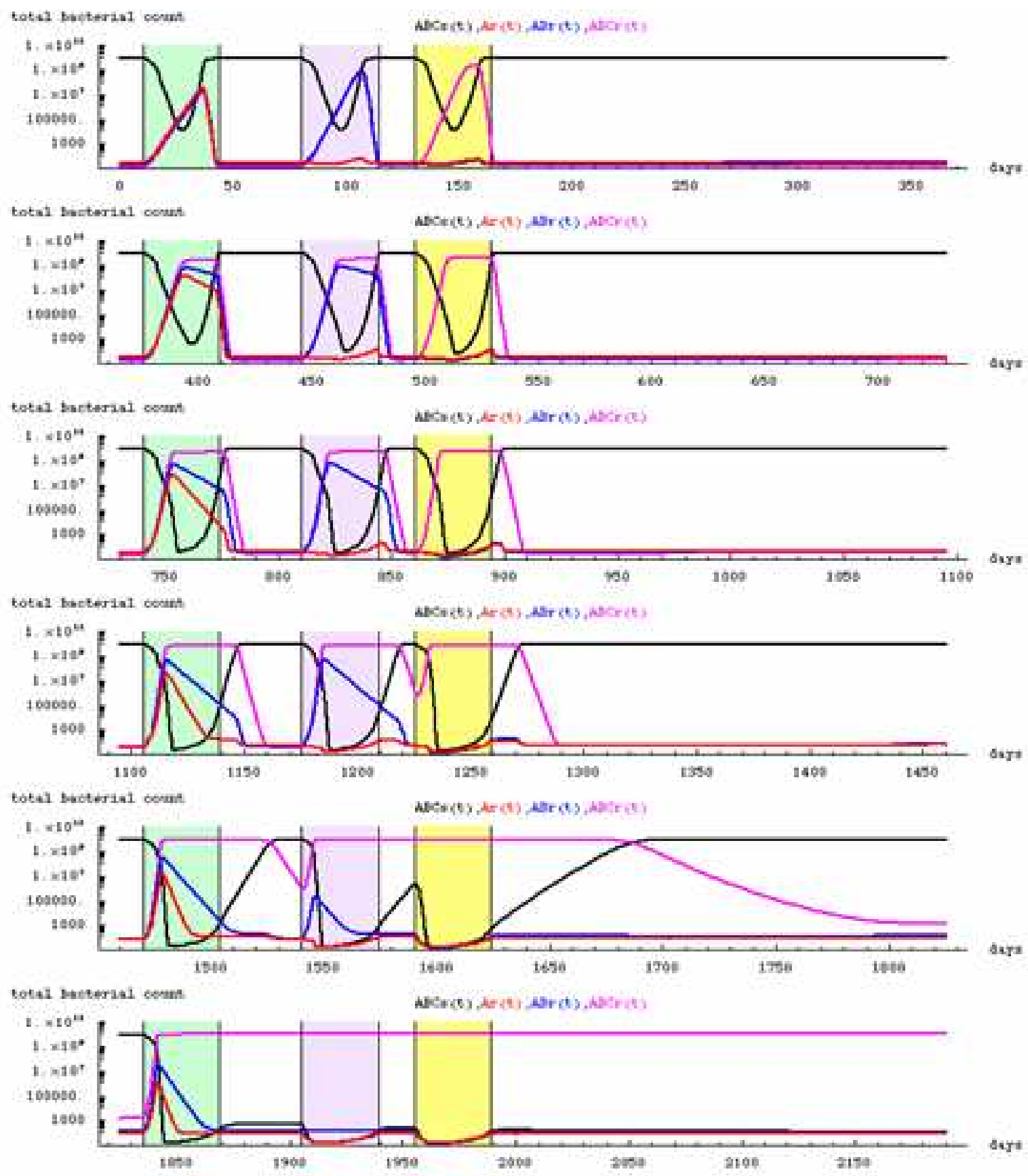

Figure 3: The total bacterial count of the 4 bacterial strains: $\mathrm{ABCs}$ (black), $\mathrm{Ar}$ (red), $\mathrm{ABr}$ (blue), and $\mathrm{ABCr}$ (magenta), as a function of time over 6 years. Three antibiotics A (green), B (purple), and $\mathrm{C}$ (yellow), are administered every 12 months, and the initial difference in bacterial fitness between resistant strains and the susceptible strain is $10 \%$. The fully resistant strain $\mathrm{ABCr}$ is dominant at the beginning of the 6th antibiotic cycle, at approximately 5 years. 


\subsection{Repetitive exposure to three-antimicrobial regimen}

With repetitive antimicrobial exposure, the bacterial fitness of the resistant strains increases over time. Since compensatory mutations occur more frequently during exposure to the antibiotic to which the strain is resistant to, the bacterial fitness increases more rapidly for the $\mathrm{ABCr}$ strain compared to the $\mathrm{Ar}$ or $\mathrm{ABr}$ strain with repetitive antibiotic exposure. The time to $\mathrm{ABCr}$ becoming established as the dominant strain occurs when the bacterial fitness of $\mathrm{ABCr}$ exceeds the bacterial fitness of $\mathrm{ABCs}, \mathrm{Ar}$ and $\mathrm{ABr}$, and the fraction of the carrying capacity $\mathrm{ABCr}$ occupies at the time this occurs (Figure 3). Although the bacterial fitness of $\mathrm{Ar}$ and $\mathrm{ABr}$ increase with antibiotic exposure, these strains do not predominate due to the limitations imposed by the finite carrying capacity of the total bacterial population and the ultimate dominance of the fully resistant strain.

Figure 2 displays the changes in bacterial fitness over 6 repeated cycles of exposure to antimicrobials A, B and C every 12 months, and Figure 3 shows the changes in total bacterial count of each of the four strains during this time. Prior to the administration of antimicrobial A in the 6th cycle, the fitness of $\mathrm{ABCs}$ is dominant (Figure 2) and the total population count of ABCs is dominant (Figure 3). The fitness of $\mathrm{ABCr}$ becomes dominant during the administration of antimicrobial A in this cycle (Figure 2), as does its total population count (Figure 3).

Model simulations demonstrated that exposing the patient to the three-antimicrobial regimen every 6 months, decreased the time of emergence of the ABCr strain as the dominant strain to 3.2 years, compared to 5.04 years if exposed every 12 months. A reciprocal increase in the time to predominance occurs if the time of repetitive cycling is increased to 24 months, whereby the $\mathrm{ABCr}$ strain dominates at 6.1 years (Figure 4).

\subsection{Bacterial fitness of resistant strains}

Reductions in fitness costs among antimicrobial-resistant bacteria through compensatory mutations allow resistant strains to have a competitive advantage over susceptible strains. To quantify the impact of harboring subpopulations of resistant strains that have greater fitness at baseline, simulations were performed with varying percentages of fitness of resistant strains relative to the susceptible strain. If the individual is exposed to the three-antimicrobial regimen every 12 months and the initial bacterial fitness of the resistant strains relative to the pan-susceptible strain is increased from $10 \%$ to $30 \%$ and $50 \%$, the time for the $\mathrm{ABCr}$ strain to dominate decreases rapidly from approximately 5 years to 3.2 years and 2.2 years, respectively. As seen in Figure 4, an increase in baseline fitness and an increase in frequency of exposure to the 3-drug regimen further decrease the time to $\mathrm{ABCr}$ domination.

\section{Discussion}

This mathematical model conceptualizes the emergence of MDRGN through the evolution of compensatory mutations, which produce biologically fitter resistant strains, and quantifies the effect of repetitive antimicrobial exposure on the time to which the MDR strains predominate over the pansusceptible, single- and two-drug resistant strains. 


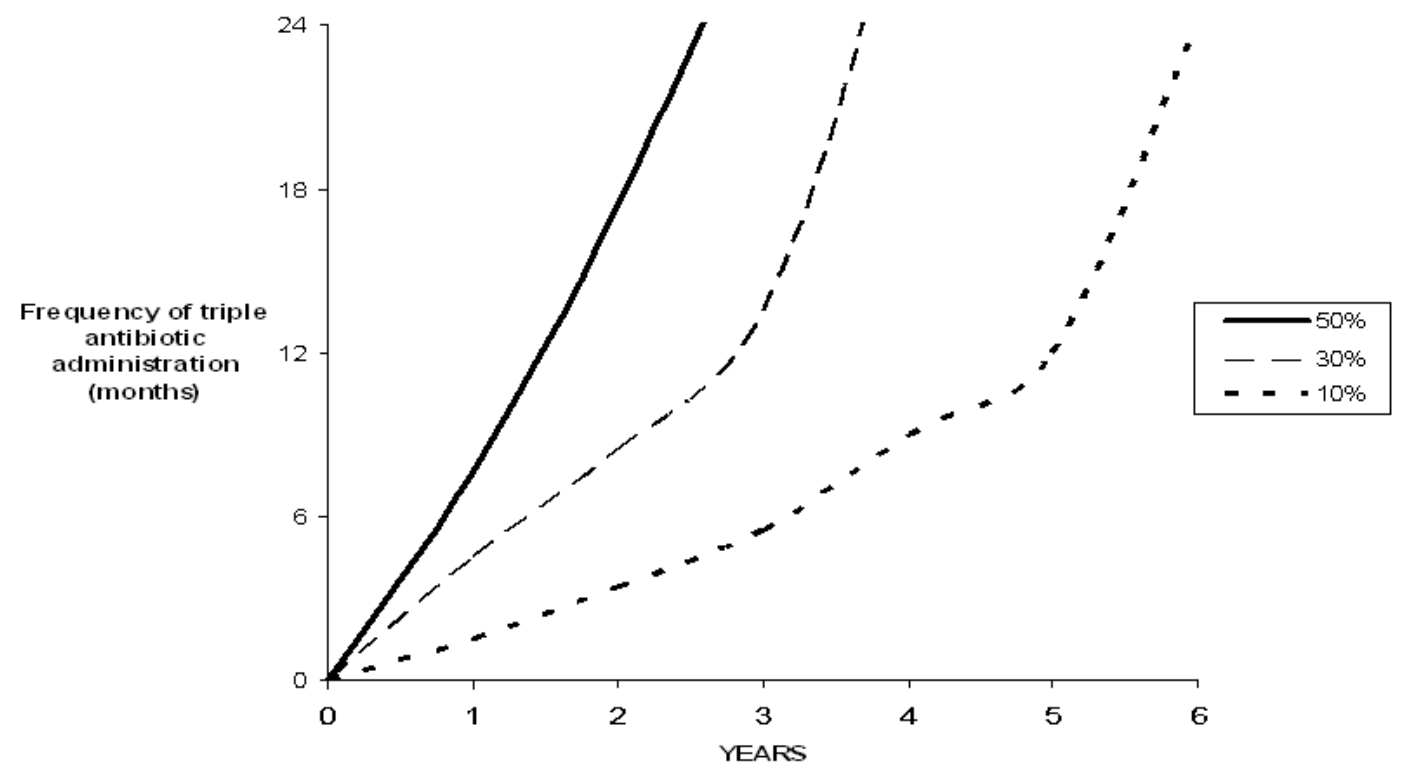

Figure 4: Time to domination of the total population count of the multidrug-resistant bacterial strain, $\mathrm{ABCr}$, with varying baseline bacterial fitness of resistant strains relative to the pansusceptible strain $\mathrm{ABCs}(10 \%, 30 \%, 50 \%)$ as a function of the frequency of administration of the 3-drug antibiotic exposure. 
The model predicts that initially, in the absence of antimicrobial exposure, the pan-susceptible strain dominates over the MDRGN, single- and two-antimicrobial resistant strains by almost 10 orders of magnitude due to the very rapid doubling time of the pan-susceptible bacteria and the initial fitness cost of the resistant strains. During treatments however, these roles are reversed for the appropriate resistant strains. Gradually over time and with repetitive antimicrobial exposure, the resistant strains gain fitness on the pan-susceptible strain, with the fully resistant strains MDRGN finally dominating all other strains, even in the absence of antimicrobial exposure. The time to MDRGN becoming established occurs when, through compensatory mutations, the bacterial fitness of the fully resistant strain exceeds the fitness of the pan-susceptible strain and proliferates to irreversible dominance. Numerous studies supporting the acquisition of fitness-compensatory mutations have been published and recently reviewed by Maisner-Patin and Andersson [1,23]. Among the most clinical relevant reductions in fitness cost associated with antimicrobial resistance include isoniazid-resistant Mycobacterium tuberculosis [33], protease inhibitors-resistant HIV-1 strains [22], azole-resistant Candida albicans [7], and clarythromycin-resistant Helicobacter pylori [4].

This model also projects that decreasing the frequency of triple antibiotic exposure substantially increases the time to emergence of the MDRGN strain. These findings emphasize the importance of reducing antimicrobial use. They also highlight the impact of repetitive exposure with different antimicrobials as a key factor in the emergence of MDRGN. Since the rate of formation of compensatory mutations is proportional to the population count of bacteria, the bacterial fitness of the MDRGN strain, which is resistant to all three antimicrobials, will increase with repetitive antibiotic exposure faster than the single- and two-drug resistant strains.

There are several important implications that can be extrapolated from this model. First, when the bacterial fitness of MDRGN exceeds that of pan-susceptible strains then there is no longer a selective advantage for susceptible strains to predominate in the absence of antimicrobial pressure. The evolution of MDRGN into strains in which resistance no longer confers any cost to fitness and which are therefore as fit or fitter than the susceptible strain implies that further reduction in antimicrobial exposure would not result in a decrease of MDRGN, since the absence of selective antimicrobial pressure would no longer favor the emergence of the susceptible strains. Second, the association between antimicrobial exposure and the emergence of MDRGN involves the complex dynamics of the competing strains and the scheduling of therapy. Analyzing the impact of antimicrobial exposure on the emergence of MDRGN strains requires the consideration of both the simultaneous and the sequential exposure to different antimicrobials, and cannot be restricted to individual antimicrobials. Third, this model implies that antimicrobial cycling would promote the emergence of MDRGN strains. The benefit of antimicrobial cycling in decreasing antimicrobial resistance remains controversial $[3,14,29,30]$. Among those studies that have shown benefit, the outcome focused on single-drug resistance. This model emphasizes the negative impact of antibiotic cycling through sequential antimicrobial exposure on the emergence of MDRGN strains.

Clinical studies to delineate the time to emergence of MDRGN would be extremely difficult to conduct, given the complexities of factors contributing to their emergence. Quantitative validation of this mathematical model is therefore limited. Nevertheless, the concepts utilized in the model are derived from fundamental principles of bacterial population dynamics and the effects of antibiotic exposure, and therefore validate the qualitative insight provided by the model. To provide an 
overall representation of the factors contributing to the emergence of MDRGN, and to simplify the interpretation of the model's conclusions, several factors were not included in the model, including the complexities of different types of bacterial resistance mechanisms among gram-negative bacteria, reversion to a susceptible phenotype and differential effects of different antibiotics on the gastrointestinal flora. Future models will need to quantify the impact on the time to emergence of MDRGN.

Evolution, by definition, favors the survival of the fittest, and all living creatures adapt to their changing environment as a means of survival. With the exponential rise in antimicrobial use throughout the world providing strong selection pressure for MDRGN, which are biologically more fit than their pan-susceptible, single- or two-drug-resistant counterparts, the rapid emergence and spread of MDRGN seems intuitive. This mathematical model provides a theoretical framework at the individual level. Structured population models, which incorporate the transmission of antimicrobial-resistant bacteria between patients, healthcare institutions and the community $[2,10,34,35]$, are the next necessary steps to successfully understand the complexities of the emergence and spread of MDRGN, and to direct effective preventive strategies.

\section{Appendix. Mathematical derivation of model}

The model consists of a nonlinear system of 4 ordinary differential equations simulating the dynamics of the 4 competing strains of bacteria in an individual patient subject to a repeated cyclic regimen of three antimicrobials. The 4 strains are ABCs (wild type - sensitive to all 3 drugs), Ar (resistant to antimicrobial $\mathrm{A}$ ), $\mathrm{ABr}$ (resistant to antimicrobials $\mathrm{A}$ and $\mathrm{B}$ ), and $\mathrm{ABCr}$ (resistant to antimicrobials $\mathrm{A}, \mathrm{B}$ and $\mathrm{C}$ ). The populations of the bacterial strains at time $t$ days are $\mathrm{ABCs}(t)$, $\operatorname{Ar}(t), \operatorname{ABr}(t)$, and $\mathrm{ABCr}(t)$, respectively, and the total population is $\mathrm{P}(t)=\mathrm{ABCs}(t)+\operatorname{Ar}(t)+$ $\mathrm{ABr}(t)+\mathrm{ABCr}(t)$. The patient undergoes the 3-drug treatment cycle A-B-C for repeatedly over years. The 4 bacterial populations satisfy the system of differential equations

$$
\begin{gathered}
\frac{d}{d t} A B C s(t)=(\beta A B C s(t)-\mu A B S s(t)-P(t) / C C) A B C s(t)+S, \\
\frac{d}{d t} A r(t)=(\beta A r(t)-\mu A r(t)-P(t) / C C) A r(t)+S, \\
\frac{d}{d t} A B r(t)=(\beta A B r(t)-\mu A B r(t)-P(t) / C C) A B r(t)+S, \\
\frac{d}{d t} A B C r(t)=(\beta A B C r(t)-P(t) / C C) A B C r(t)+S,
\end{gathered}
$$

with initial conditions $A B C s(0), A r(0), A B r(0)$, and $A B C r(0)$ given at time $t=0$.

The explanation of the terms in equations (5.1)-(5.4) is as follows:

(A.1) The number of generations per day $(G P D)$ of the wild strain is constant at $G P D=7$. The intrinsic fitness $(\beta A B C s)$ of the wild strain is $(\log 2)(\mathrm{GPD})$, which means the wild strain has a doubling time of $24 / G P D$ hours if growth is unconstrained by a limiting carrying capacity. The intrinsic doubling time of $\mathrm{ABCs}$ is constant at $\log 2 / \beta A B C s=\log 2 / 4.85$ days $=3.43 \mathrm{hr}[8,36]$. 
(A.2) The carrying capacity is shared by the 4 strains and the effect of the limited carrying capacity is modeled in each equation by the loss rate $-P(t) / C C$, where $C C=10^{10}$ is the carrying capacity parameter.

(A.3) The treatment regimen in the first year is as follows: antimicrobial A begins at day 10, reaches peak concentration after 7 days, maintains peak concentration for 7 more days, and is eliminated 20 days post exposure. In a similar way antimicrobial B begins at day 80 and antimicrobial $\mathrm{C}$ begins at day 130. Each drug results in a proportional loss of the appropriate drug-sensitive populations during the 14 day treatment exposure and 20 day post-exposure periods with loss rates $-A B C s(t),-A r(t),-A B r(t)$. These loss rates day increase linearly from 0 to the maximum 6 during the first 7 days of exposure, then remain constant for 7 more days, and finally decrease linearly back to 0 after 20 more days. The parameters are chosen to reduce the drug sensitive populations to very low levels in 2 days. The cycle is repeated each year for 6 years.

(A.4) The intrinsic fitness $\beta A B C s=(\log 2) G P D$ of the wild strain is constant, but the intrinsic fitness $(\beta A r(t), \beta A B r(t), \beta A B C r(t))$ of the resistant strains are increasing functions of time. At time 0 , the resistant strains have fitness $\beta A r(0)=\beta A B r(0)=\beta A B C r(0)=.1 \beta A B C s$. This increase is on-going at a constant rate, but is even higher during treatments and for a period of time after treatments. For the Ar strain the on-going rate of fitness increase is $.0002 \beta A r(0) / \log 2 /$ day. During the 14 days of antimicrobial A exposure and for an additional period of 20 days, there is an additional increase in fitness given by $.0005 \beta A r(0) / \log 2 /$ day. Similar terms account for the increase in fitness for the $\mathrm{ABr}$ and $\mathrm{ABCr}$ strains.

(A.5) There is a small background constant source $S=100 /$ day for each of the 4 strains imputed each day due to environmental conditions or host mutations.

The system (5.1)-(5.4) is solved numerically using MATHEMATICA (Wolfram Research, Champaign, IL). The program code is available upon request.

\section{Acknowledgments}

GW was supported by National Science Foundation Grant DMS-0518576. MAH was supported by the National Science Foundation. Any opinion, findings, and conclusions or recommendations expressed are those of the authors and do not necessary reflect the views of the National Science Foundation.

\section{References}

[1] D.I. Andersson. Persistence of antibiotic resistant bacteria. Curr. Opinion Microbiol. 6 (2003), 452-456.

[2] D.J. Austin, K.G. Kristinsson, R.M. Anderson. The relationship between the volume of antimicrobial consumption in human communities and the frequency of resistance. Proc. Natl. Acad. Sci. U S A. 96 (1999), 1152-1156. 
[3] C.T. Bergstrom, M. Lo, M. Lipsitch. Ecological theory suggests that antimicrobial cycling will not reduce antimicrobial resistance in hospitals. Proc. Natl. Acad. Sci. USA. 101 (2004), 13285-13290.

[4] B. Bjorkholm, M. Sjolund, P.G. Falk, O.G. Berg, L. Engstrand, D.I.Andersson. Mutation frequency and biological cost of antibiotic resistance in Helicobacter pylori. Proc. Natl. Acad. Sci. U S A. 98 (2001), 14607-14612.

[5] M.J. Blaser. The genetic gymnastics of our indigenous microbes. N. Engl. J. Med. 346 (2002), 2083-2085.

[6] J.E. Bouma, R.E. Lenski. Evolution of a bacteria/plasmid association. Nature. 335 (1988), 351-352.

[7] L.E. Cowen, D. Sanglard, D. Calabrese, C. Sirjusingh, J.B. Anderson, L.M. Kohn. Evolution of drug resistance in experimental populations of Candida albicans. J. Bacteriol. 182 (2000), 1515-1522.

[8] A.J. Cullum, A.Bennett, R.E.Lenski. Evolutionary adaptation to temperature. IX. Preadaptation to novel stressful environments of Escherichia coli adapted to high temperature. Evolution. 55 (2001), 2194-2202.

[9] E.M.C. D’Agata. Rapidly Rising Prevalence of Nosocomial Multidrug-Resistant GramNegative Bacilli: A 9-Year Surveillance Study. Infect. Control Hosp. Epidemiol. 25 (2004), 842-846.

[10] E.M.C. D’Agata, M.A .Horn, G. Webb. Transmission dynamics of vancomycin-resistant enterococci in hemodialysis units using mathematical modeling. J. Infect. Dis. 185 (2002), 766-773.

[11] E.M.C. D'Agata, L. Venkataraman, P. DeGirolami, M. Samore. The molecular epidemiology of acquisition of ceftazidime-resistant gram-negative strains in a non-outbreak setting. J. Clin. Microbiol. 35 (1997), 2602-2605.

[12] L. Edelstein-Keshet. Mathematical models in Biology. 1st ed. Boston, MA, McGraw Hill. 1988, 115-163.

[13] A.C. Gales, R.N. Jones, J.J. Turnidge, R. Rennie, R. Ramphal. Characterization of Pseudomonas aeruginosa isolates: occurrence rates, antimicrobial susceptibility patterns, and molecular typing in the global SENTRY antimicrobial surveillance program, 1997-1999. Clin. Infect. Dis. 32 (2001), 133-145.

[14] D. Gruson, G. Hilbert, F. Vargas, R. Valentino, C. Bebear, A. Allery, C. Bebear, G. GbikpiBenissan, J.P. Cardinaud. Rotation and restricted use of antibiotics in a medical intensive care unit. Impact on the incidence of ventilator-associated pneumonia caused by antibioticresistant gram-negative bacteria. Am. J. Respir. Crit. Care Med. 162 (2000), 837-843. 
[15] J.A. Karlowsky, D.C. Draghi, M.E. Jones, C. Thronsberry, I.R. Friedland, D.F. Sahm. Surveillance for antimicrobial susceptibility among clinical isolates of Pseudomonas aeruginosa and Acinetobacter baumannii from hospitalized patients in the United States, 1998 to 2001. Antimicrob. Agents Chemother. 47 (2003), 1681-1688.

[16] J.A. Karlowsky, M.E. Jones, C. Thornsberry, I.R. Friedland, D.F. Sahm. Trends in antimicrobial susceptibilities among Enterobacteriaceae isolates from hospitalized patients in the untied States form 1998 to 2001. Antimicrob. Agents Chemother. 47 (2003), 1672-1680.

[17] M. Lipsitch, B.R. Levin. The population dynamics of antimicrobial chemotherapy. Antimicrob. Agents Chemother. 41 (1997), 363-373.

[18] R.E. Lenski, M.R. Rose, S.C. Simpson, S.C. Tadler. 1991. Long-term experimental evolution in Escherichia coli. Adaptations and divergence during 2,000 generations. Amer. Nat. 138 (1991), 1315-1341.

[19] R.E. Lenski, M. Travisano. Dynamics of adaptation and diversification: a 10,000generation experiment with bacterial populations. Proc. Natl. Acad. Sci. U S A. 91 (1994), 6808-6814.

[20] B.R. Levin, M. Lipsitch, V. Perrot, S. Schrag, R. Antia, L. Simonsen, N.M. Walker, F.M. Stewart. The population genetics of antibiotic resistance. Clin. Infect. Dis. 24 (1997), 9-16.

[21] B.R. Levin, V. Perrot, N. Walker. Compensatory mutations, antibiotic resistance and the population genetics of adaptive evolution in bacteria. Genetics 154 (2000), 985-997.

[22] M.F. Maguire, R. Guinea, P. Griffin, R.C. Elston, J. Wolfram, N. Richards, M.H. Hanlon, D.J. Porter, T. Wrin, N. Parkin, M. Tisdale, E. Furfine, C. Petropoulos, B.W. Snowden, J.P. Kleim. Changes in human immunodeficiency virus type 1 Gag at positions L449 and P453 are linked to I50V protease mutants in vivo and cause reduction of sensitivity to amprenavir and improved viral fitness in vitro. J. Virol. 76 (2002), 7398-7406.

[23] S. Maisnier-Patin, D.I .Andersson. Adaptation $f$ the deleterious effects of antimicrobial drug resistance mutations by compensatory evolution. Research Microbiol. 155 (2004), 360-369.

[24] National Nosocomial Infections Surveillance System. National Nosocomial Infections Surveillance (NNIS) System Report, data summary from January 1992 through June 2003, issued August 2003. Amer. J. Infect. Control.31 (2003), 481-498.

[25] B. Ortega, J. Groeneveld, C. Schultsz. Endemic multidrug-resistant Pseudomonas aeruginosa in critically ill patients.Infect. Control Hosp. Epidemiol. 25 (2004), 825-831.

[26] E. Paramythiotou, J.C. Lucet, J.F. Timsit, D. Vanjak, C. Paugam-Burtz,J.L. Trouillet, S. Belloc, N. Kassis, A. Karabinis, A. Andremont. Acquisition of multidrug-resistant Pseudomonas aeruginosa in patients in intensive care units: role of antibiotics with antipseudomonal activity. Clin. Infect. Dis. 38 (2004), 670-677. 
[27] I. Pelupessy, M.J. Bonten, O. Diekmann. How to assess the relative importance of different colonization routes of pathogens within hospital settings.Proc. Natl. Acad. Sci. USA 99 (2002), 5601-5605.

[28] A. Pop-Vicas, E.M.C. D'Agata. The Rising Influx of Multi-Drug Resistant Gram-Negative Bacteria into a Tertiary Care Hospital. Clin. Infect. Dis. 40 (2005), 1792-1798.

[29] L.A. Puzniak, J. Mayfield, T. Leet, M. Kollef, L.M. Mundy. Acquisition of vancomycinresistant enterococci during scheduled antimicrobial rotation in an intensive care unit. Clin. Infect. Dis. 33 (2001), 151157.

[30] D.P. Raymond, S.J. Pelletier, T.D. Crabtree, et al. Impact of a rotating empiric antibiotic schedule on infectious mortality in an intensive care unit.Crit. Care Med. 29 (2001), 11011108 .

[31] D.S. Reeves. The effect of quinolone antibacterials on the gastrointestinal flora compared with that of other antibacterials.J. Antimicrob. Chemother. 18 (1986), 89-102.

[32] S.F. Schragg, V. Perrot. Reducing antibiotic resistance. Nature 381 (1996), 120-121.

[33] D.R. Sherman, K. Mdluli, M.J. Hickey, T.M. Arain, S.L. Morris, C.E. Barry, C.K. Stover. Compensatory ahpC gene expression in isoniazid-resistant Mycobacterium tuberculosis. Science. 272 (1996), 1641-1643. .

[34] D.L. Smith, J. Dushoff, E.N. Perencevich, A.D. Harris, S.A. Levin. Persistent colonization and the spread of antibiotic resistance in nosocomial pathogens: resistance is a regional problem. Proc. Natl. Acad. Sci. U S A. 101 (2004), 3709-3714.

[35] D.L. Smith, S.A. Levin, R. Laxminarayan. Strategic interactions in multi-institutional epidemics of antibiotic resistance. Proc. Natl. Acad. Sci. U S A. 102 (2005), 3153-3158.

[36] L.M. Tamagnini, R.D.J. Gonzalez. Bacteriological stability and growth kinetics of Pseudomonas aeruginosa in bottled water. Appl. Microbiol. 83 (1997), 91-94.

[37] E.J. Vollaard, H.A.L. Clasener. Colonization resistance. Antimicrob. Agents Chemother. 38 (1994), 409-414. 